by acceptance of this article, the publisher or recipient acknowledges the U.S. Governmicni's right to retain a nonexclusive, royalty free license in and to anv copyright covoring the article

\section{$\operatorname{CON}-820345--3$ \\ CONF-8.20.345--3 \\ DE82 011967}

\title{
ION CYCLOTRON HEATING EXPERIMENTS IN EBT-S*
}

F. H. Baity, h. in. Laviz, O. C. Elciridoc, '. C. Glowienka, D. L. Hillis, D. A. kasmussen, and R. K. Richards

Oak: Ridge National Laboratory

Oak Ridge, Tennessee 37830 USA.

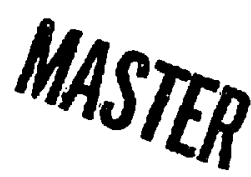

J. H. Pullen and T. L. Grens

MoDonnell Douglas Astronautics Company

St. Louis, Nissouri ó3166 …

Atstract

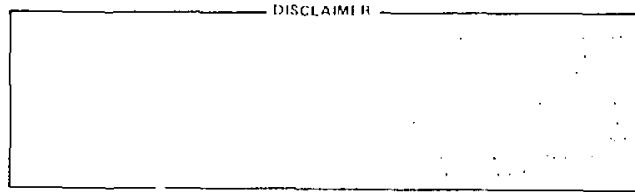

Fast-wave heating and propagation experiments in the ion cyclotron range of frequencies ( ICRF) have been carried out on ELf-S under steady-state conditions at power levels up to $20 \mathrm{ki}$ and under pulsed conditions at power levels up to $100 \mathrm{~kW}$. The waves were launched using a single Faraday-shielded loop antenna installed on the midplane of one of the 24 cavity sectors. Substantial ion heating was observed at frequencies above the second harmonic for hydrogen plasmas and at frequencies above the third harmonic for deuterium plasmas, corresponding with the onset of wave propagation around the torus. The heating under these conditions is anomalous. In the case of deuterium plasmas, a small residual coneentration (2-5\%) of hydrogen was heated, but this component did not appear to affect the jeuterium heating.

\section{Introductio:}

Experiments rith ICRE reating on EBT-S word uertaken to provide the physics and technology base for application oi fast-wave ion heating on the next-generation proof-ô-principis EEI experiment,

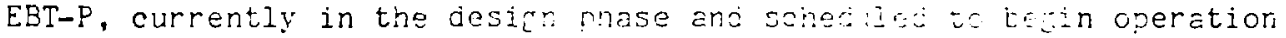
in the mid-1980's. fuxiliar: ion heatire $: \cdots, \cdots$ order to understand ion behavion in EBT geometry and is orjer to attain plasma beta values of approximately 5\%. The size and parameters of the

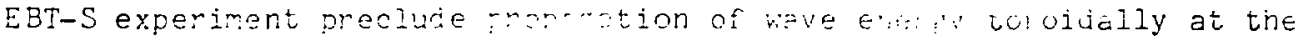
fundamental frequency of harcon, so that minorit $\because$ heasing in the two-ion hybrid regime camo- ie expected outsiae tise aniona cavity. Toroidal wave propagation in EST-S occurs at frejucicies above the second harmonic frequency of hyrogen, where bus daning by the plasma ions is expected to be siaii. iave proparacion : aji-f will be much

\footnotetext{
* Research sponsored by the ofrice of Fusion Eneruy, U. S. Department of Energy unier contrect $\because-7 ! n=-5 n=-26$ with the "ason Corbide

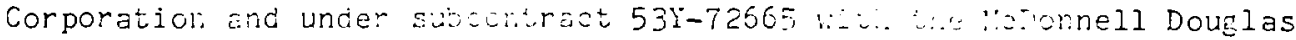
Astronadic: Eoripen.
} 


$$
-2-
$$

improved, with several modes present at the fundamental frequency for ilyirogen.

The EBT-S experiments are conducted steady-state so that active cooling of the antenna and Faraday shield is necessary. In addition the plasma in EBT-S is formed by electron cyclotron heating, so that the antenna is required to be compatible with a high level of nicrowave radiation.

This paper describes unly the ion heating results obtained with charge-exchange analyzers. Power coupling and wave propagation are described in another paper in this session [1].

\section{Description of Apparatus}

The installation of the ICRH antenna is shown schematicaliy in Fik. 1. The antenna covers a poloidal angle of $120^{\circ}$, is water-cooled,

Fig. 1. Schematic drawing or the antenna and matching circuit installation on EBT-S. The vacuum variable capacitors are remotely tuned during operation. The antenna is mounted in a modified cavity sector midway between two toroidal magnetic field coils. 
and is surrounded by a double aluminum Faraday shield, which is also water-cooled for steady-state operation. The antenna connects to a broadband matching circuit consisting of three remotely-tuned vacuum variable capacitors, which cover the frequency range of 15-50 $\mathrm{MHz}$. The two large "parallel" capacitors are also water-cooled to permit steady-ztate operation. The present system is limited to $20 \mathrm{~kW}$ steady state in the T-mode by the Faraday shield cooling. A new Faraday shield with improved cooling has been designed which will allow operation at $100 \mathrm{~kW}$ steady state. The coax line to the matching circuit comes from an array of $r f$ power sources, including a $20-\mathrm{kW} \mathrm{cW}$, 2-30 $\mathrm{MHz}$ transmitter, a 100-kW cw, 5-30 $\mathrm{MHz}$ transmitter, and a $1.5-\mathrm{kW} \mathrm{cw}$ broadband amplifier covering the entire frequency range of the system. The best power coupling in the range of the high power transmitters occurs at $30 \mathrm{MHz}$. Therefore, measurements of heating as a function of power were conducted at $30 \mathrm{MHz}$, primarily, and measurements as a function of frequency were made with several hundred watts coupled to the plasma.

\section{Ion Heating Results}

In the absence of direct ion heating, the ions in EBT-S gain energy by collisions with plasma electrons or by some anomalous process. Since the best estimate of the neutral particle density from spectroscopy is $2 \times 10^{10} \mathrm{~cm}^{-3}$, the average ion temperature is limited by charge-exchange to $\sim 10 \mathrm{eV}$. However, in the $\mathrm{T}$-mode of operation, when the stabilizing relativistic electron rings are present, one or more high-energy tails is always observed with the charge-exchange analyzers. These tails increase in density as the TM-transition, which is the low pressure limit of the T-mode, is approached. The tails are almost nonexistent in the cold, high-density C-mode (without rings). Recently, highly resolved $H_{a}$ spectra have shown the existence of high energy neutral $\mathrm{H}$ atoms, arising from charge exchange. These spectra show a two-component ion energy distribution, with a bulk temperature of $-10-15 \mathrm{eV}$ and a tail at $60-75 \mathrm{eV}$ represnnting typically $10^{\circ}$ of the core plasma ions. This high energy tail is also measured with the charge-exchange analyzers, which sometimes observe another tail at several hundred $\mathrm{eV}$, but at a still lower percentage of the total ions. A comparison of the medium energy tail densities calculated from spectroscopy and charge-exchange gives an absolute calibration for the charge-exchange density measurements, provided the plasina ions are isotropic. The source of these high energy tails is unknown.

The addition of ICRF heating results in a substantial enhancement of both the density and temperature of the high energy tail components, with little, if any, change in the bulk ion temperature. This is a consequence of the fact that the ion energy lifetime is considerably shorter than the ion-ion energy equilibration time under normal EBT-S conditions. High energy ion tails are produced even in the C-mode during ICRF heating. In the C-mode the antenna loading is higher than in the T-mode (70\% power coupling to the plasma at $30 \mathrm{MHz}$ for a typical C-mode condition compared to $45 \%$ coupling in the middle of the T-mode with $100 \mathrm{~kW}$ of $\mathrm{ECH}$ ). Tris is presimably due both to the increased 
density in the C-mode and to the proximity of the plasma to the antenna in the absence of relativistic electron rings.

The temperature and density of the high energy tail increases with increasing amounts of ICRF heating, as shown in Fig. 2 for a $95 \%$ deuterium plasma in the $\mathrm{T}$-mode at $30 \mathrm{MHz}$, measured four cavities $\left(60^{\circ}\right.$ toroidally) from the antennas. The plasma parameters for this data were: a magnetic field strength on axis in the cavity midplane of $0.725 \mathrm{~T}(1.4 \mathrm{~T}$ in the mirror throats), an $\mathrm{ECH}$ power of $100 \mathrm{~kW}$ at 28 $\mathrm{GHz}$, and a background pressure of $1 \times 10^{-5}$ Torr. The straight 1 ines represent Maxwellian fits to the experimental data points. This graph was produced by assuming that the measured flux was due entirely to deuterons with little proton contribution. Mass analysis of the charge-exchange flux carried out using another charge-exchange analyzer. located on the antenna cavity, indicated that protons indeed contribute little for energies below $\sim 1.5 \mathrm{keV}$.

Fig. 2. Experimental distrioution functions as a function of ion energy and least-squares Maxwellian fits for several different. ICRF heating power levels. The fitted portion of each spectrum revresents a tail on a much lower energy bulk ion temperature distribution. This data was obtained with a charge-exchange analyzer four cavities from the antenna for a deuterium plasma in the $T$-mode. 
The calculation of the density of this high energy tail distribution is complicated by the fact that the energetic ions may be very anisotropic. The charge-exchange analyzers are all oriented to measure ions with only perpendicular energy. Without detailed knowledge of the pitch angle distribution of the ions, the density cannot be explicitly calculated from the charge-exchange data, but can be expressed in terms of $T_{\|}$. Analysis of the data shown in Fig. 2 in this manner is plotted in Fig. 3. Note that the apparent tail density increases rapidly as the applied ICRF heating power is increased, while the tail temperature increases only moderately.

Measurements of ion heating as a function of applied frequency were carried out with $300 \mathrm{~W}$ coupled into the plasma. The somewhat surprising result was that the heating increased steadily as the frequency was raised to the maximum frequency of the matching circuit. At $50 \mathrm{MHz}$ only the third and higher harmonics of hydrogen and the fifth and higher harmonics of deuterium are present in the plasma. Fig. 4 shows the several-fold increase in the raw charge-exchange signa? four cavities from the antenna as the applied frequency was increased to

Fig. 3. Calculated tail temperatures and densities from the data shown in Fig. 2. The mesure density is a function of the anisotropy of the tail component, thus $t i 20$ dependence on $T_{\|}^{-1 / 2}$ indicated in the right-hand ordinate. 
Fig. 4. Uncorrected charge-exchange signal as a function of energy for different applied ICRF heating frequencies, taken with thic charge-exchange analyzer four cavities from the antenna. This data was obtained with a deuterium plasna in the T-mode. No enhancement of the charge-exchange signal is observed until the onset of toroidal wave propagation which occurs at about $30 \mathrm{MHz}$ under these conditions.

$50 \mathrm{MHz}$. The threshold frequency of $30 \mathrm{MHz}$ corresponds to the onset of wave propagat.. on around the torus as measured by $\mathrm{rf}$ loop probes in several cavities. The heating mechanism responsible for th+s behavior is not yet understood. The complete EBI geometry, with strong magnetic field gradients, both radially and toroidally, makes theoretical modeling quite difficult. 


\section{Effects of ICRF Heating on Other Plasma Parameters}

The effects of ICRF heating on other core plasma parameters are slight. No change has been observed in either the electron temperature or impurity concentrations. A slight reduction in the core plasma density ( $10-15 \%)$ was observed with $15-20 \mathrm{~kW}$ of ICR $\bar{r}$ heating, as was a slight reduction in the ambipolar potential well depth. The major effect besides the observed ion heating has been an eruhancement in the relativistic electron ring stored energy. The ring energy has been doubled at $20 \mathrm{~kW}$ of ICRF heating. The source of this ring enhancement is not understood.

\section{Conclusions}

Fast-wave ICRF heating has been demonstrated to be an erfective method of incrasing the ion energy in EBT-S. Ions several cavities from the single antenna are heated when wave propagation occurs. The hesting improves with increasing frequency up to the limit of the matching circuit. This heating at high harmonics of the ion cyclotron frequency is anomalous. In addition, anomalous increases in the total stored energy of the relativistic electron rings is observed during ICRF heating. Further studies at higher power levels are planned.

\section{References}

1. J. H. Mullen, T. L. Owens, and A. L. Henderson, McDonnell Douglas Astronautics Company; F. W. Baity and O. C. Eldridge, Oak Ridge National Laboratory, "Fast-Wave Ion Cyclotron Wave Propagation and Coupling Experiments on Elmo Bumpy Torus," Third Joint Varenna-Grenoble International Symposium, Grenoble, France, March 22-27, 1982. 


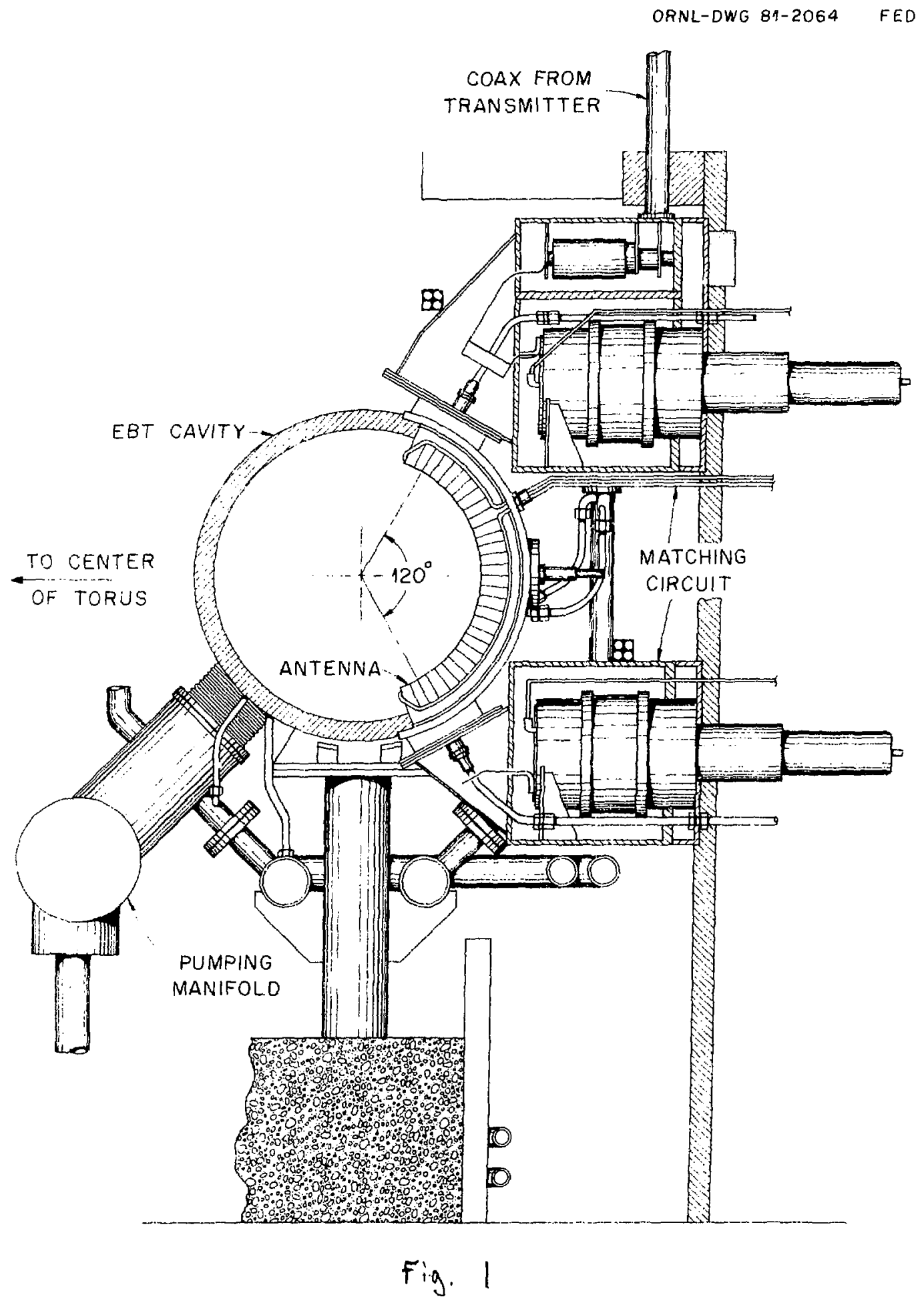




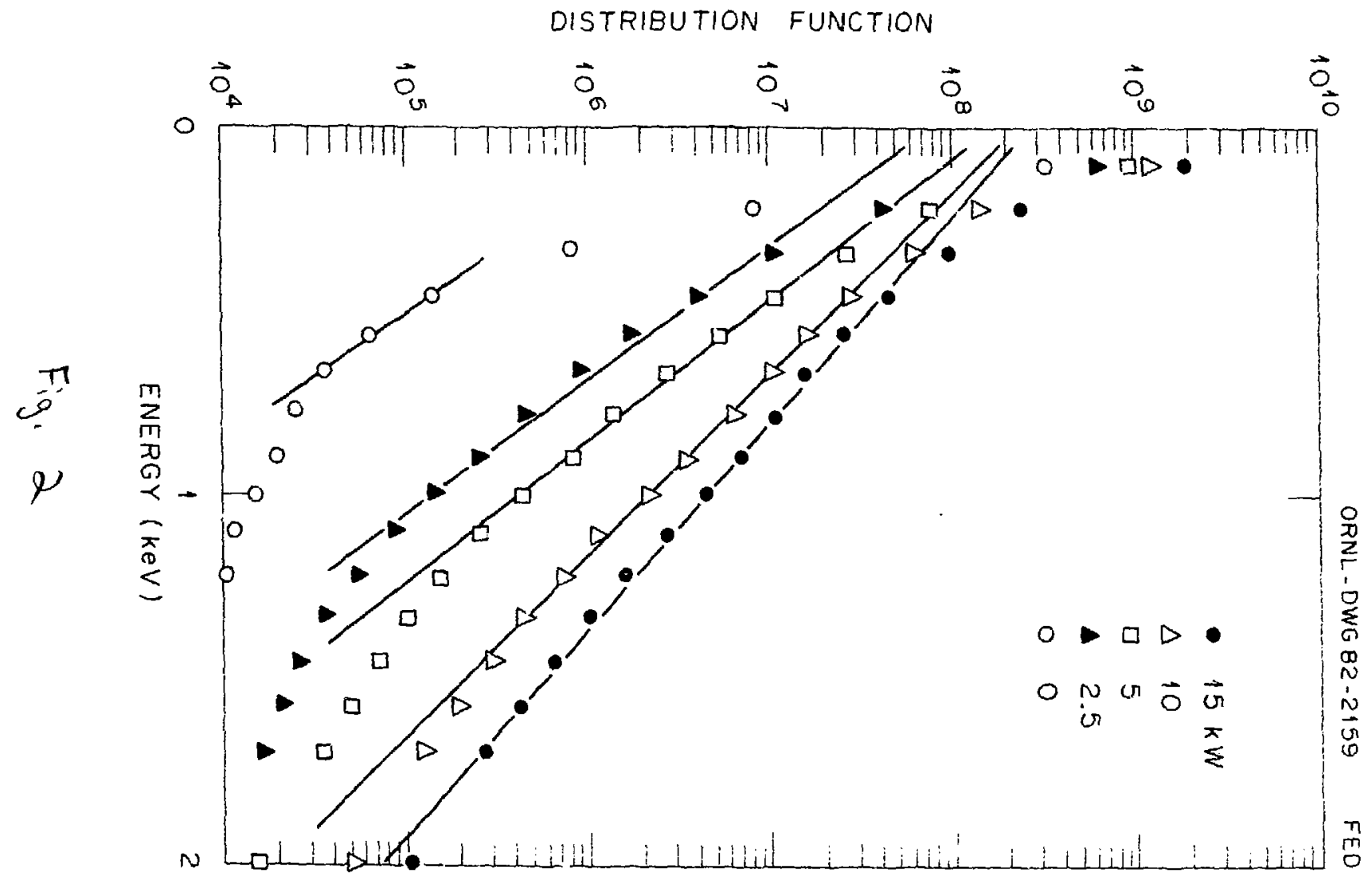




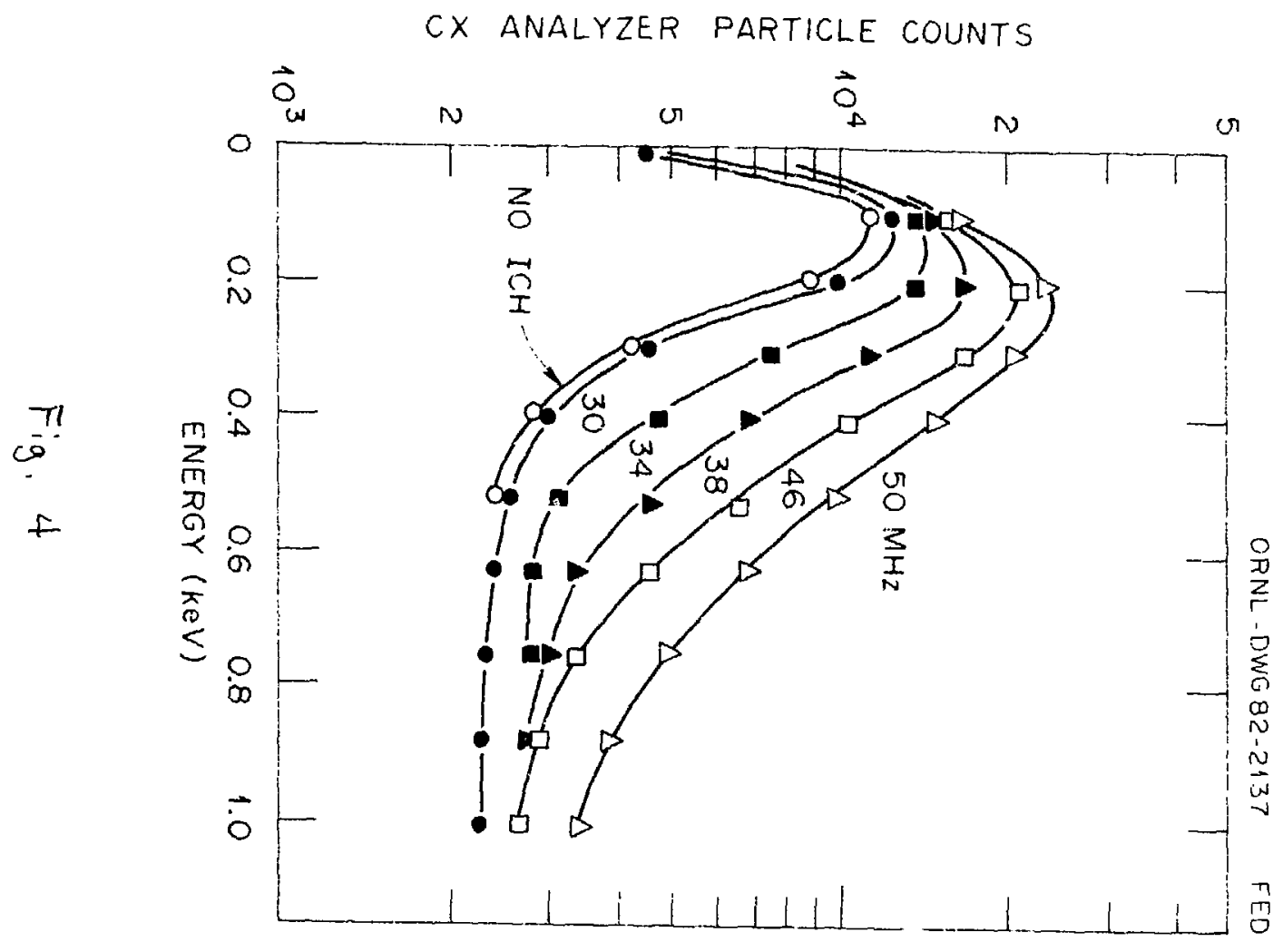

